\title{
A Multidisciplinary Approach to a Complicated Acute Paradoxical Embolism via a Patent Foramen Ovale
}

\author{
Ian Diebels ${ }^{\mathrm{a}, \mathrm{b}}$, f, Tim Wessels ${ }^{\mathrm{c}, \mathrm{d}}$, Johan Goovaerts ${ }^{\mathrm{d}}$, Michael Schreurs ${ }^{\mathrm{e}}$, Catherine Gorris ${ }^{\mathrm{e}}$, \\ Philippe De Vleeschauwer ${ }^{\text {a }}$, Marc Dubois ${ }^{\mathrm{a}}$
}

\begin{abstract}
We report a case of a 43-year-old male with a history of migraine with aura and a heterozygous factor V Leiden deficiency presenting with a complicated paradoxical arterial embolism of a deep vein thrombosis via a patent foramen ovale (PFO). Recovery was complicated with a dropfoot due to a reperfusion compartment syndrome. Treatment involved a multidisciplinary approach including embolectomy, fasciotomy, percutaneous PFO closure and extensive rehabilitation. At 5-month follow-up, the patient reported significant improvement in pain, sensation, strength and function of his leg. Repeat electromyography showed reinnervation potentials and improvement of maximal contraction in muscles innervated by the tibial and peroneal nerve, where the latter was not fully restored; however, the patient was able to walk independently again without orthosis and had restarted working. In conclusion, a complicated paradoxical embolism should be treated in a multidisciplinary setting to optimize diagnostic approach, treatment and rehabilitation.
\end{abstract}

Keywords: Paradoxical embolism; Thrombectomy; Patent foramen ovale; Factor V Leiden; Compartment syndrome; Peroneal neuropathy

\section{Introduction}

A paradoxical embolism is a form of venous embolization leading to an acute arterial occlusion due to cardiac or pulmonary shunting. The presence of a deep vein thrombosis (DVT)

Manuscript submitted August 21, 2017, accepted August 28, 2017

aDepartment of Vascular and Thoracic Surgery, Heilig-Hartziekenhuis, Lier, Belgium

${ }^{b}$ Faculty of Medicine and Health Sciences, University of Antwerp, Edegem, Belgium

'Faculty of Medicine, Department of Internal Medicine, University of Leuven ${ }^{\mathrm{d} D e p a r t m e n t ~ o f ~ C a r d i o l o g y, ~ H e i l i g-H a r t z i e k e n h u i s, ~ L i e r, ~ B e l g i u m ~}$

eDepartment of Physical Medicine and Rehabilitation, Heilig-Hartziekenhuis, Lier, Belgium

${ }^{f}$ Corresponding Author: Ian Diebels, Department of Vascular and Thoracic Surgery, Heilig-Hartziekenhuis, Lier, Mechelsestraat 24, 2500 Lier, Belgium. Email: ian.diebels@gmail.com

doi: https://doi.org/10.14740/jmc2897w and right-to-left shunting is mandatory for the diagnosis [1]. With a prevalence of up till $25 \%$, a patent foramen ovale (PFO) is a common cardiac malformation $[2,3]$. In the presence of a $\mathrm{PFO}$, the incidence of peripheral arterial embolization ranges from $4.5 \%$ to $8 \%$ [4-6]. In this report, we describe the complex diagnostic and treatment of a paradoxical embolization of a DVT through a PFO due to an acute right-to-left shunt following massive pulmonary embolism, complicated by compartment syndrome in two compartments of the lower leg with severe peroneal and tibial neuropathies.

\section{Case Report}

A 43-year-old male was admitted to the emergency department with symptoms of progressive cramping, non-radiating pain in the left lower limb and foot weakness for 2 days. His medical history included migraine with visual and sensitive aura (hemihypoesthesia, hemiparesthesia, and loss of proprioception mostly in the right side of the body) since the age of 12 . He was treated with propranolol $40 \mathrm{mg}$ once daily, and the actual frequency of the attacks which could last for 2 days was significantly reduced to 2 - 3 times a year. Two weeks before admission, he started to experience increased exercise-induced dyspnea.

Initial clinical examination revealed an anemic cold left foot up till the level of the ankle, a prolonged capillary refill (>3 s) and weak pulsation on the ipsilateral femoral level with absence of pulsations on the popliteal level. Neurological examination showed a unilateral paralysis of ankle and toe dorsiflexion, combined with paralysis of ankle plantar flexion, pretibial hypoesthesia and a reduced Achilles-reflex. Clinical examination of the contralateral leg and further systemic evaluation revealed no abnormalities.

Doppler examination demonstrated no signal distal from the ipsilateral groin. Duplex ultrasound examination of the ipsilateral leg revealed a normal triphasic flow on the level of the iliac and common femoral arty (CFA), but complete occlusion of the superficial femoral artery (SFA) and deep femoral artery (DFA). However, on the level of the popliteal artery (p2-p3) distally weak monophasic could still be found.

An emergency embolectomy was performed. The patient was placed in supine position. The left femoral bifurcation was exposed via a longitudinal incision in the ipsilateral groin. After clamping of the individual femoral vessels, the DFA and 


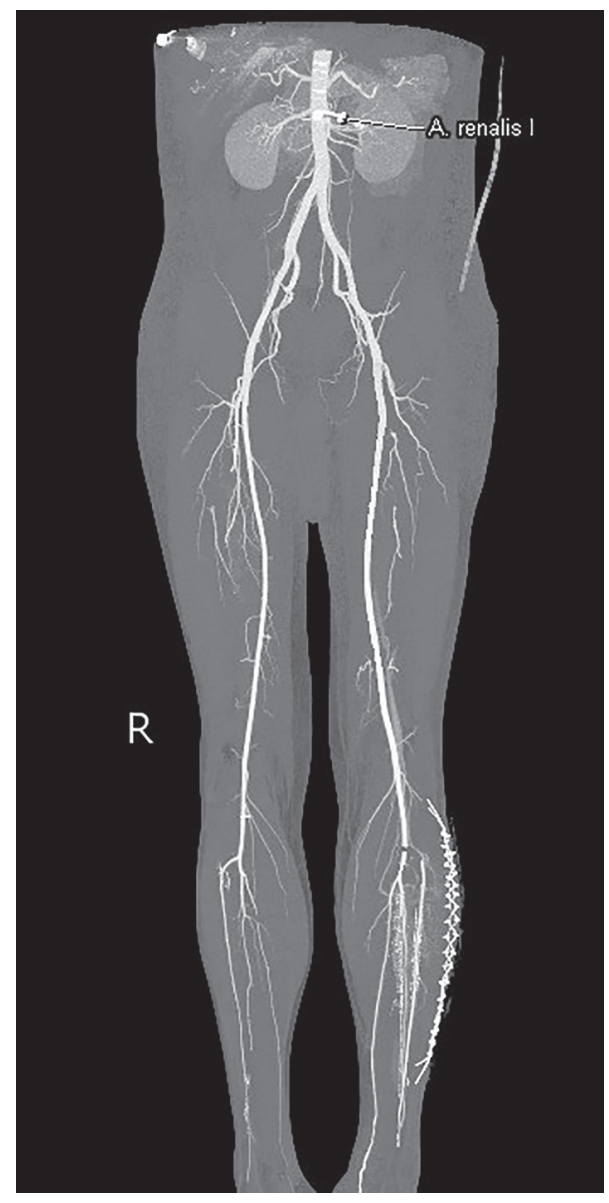

Figure 1. Computed tomography angiography of the abdomen and lower extremities. Successful revascularization with only a minor occlusion at the ipsilateral ostium of the anterior tibial artery.

SFA were opened via a longitudinal arteriotomy. A thrombectomy of both vessels was performed via the insertion of a Fogarty thrombectomy catheter. The accessory vein was resected to allow for an autologous patchplasty of the arteriotomy. A drain was placed and the wound was closed in multiple layers. Postoperative evaluation revealed a normal coloured ipsilateral foot with normal capillary refill; however, pulsations of the dorsal pedal artery or posterior popliteal artery remained absent. A thick non-constricting cotton wool bandage was applied and the patient's bed was placed in an anti-Trendelenburg position. On the first postoperative day, clear pulsations were found over both DPA and PTA; however, the neurologic deficits remained. Prophylactic low molecular weight heparine was administered subcutaneously.

Because of neurologic symptoms and progressive disproportional pain, exploration of the lower leg was planned suspecting acute compartment syndrome (ACS). After a lateral longitudinal incision of the left lower leg, a clear bulging of the anterior and (antero)lateral compartment was observed. After the fasciotomy of these two compartments, the initial anemic appearance of the anterior compartment slowly resolved within several minutes. A shoelace technique suture with skin staples was created to allow for a delayed closure.
CT-angiogram of the abdomen and lower extremities on day 4 postoperatively revealed a successful revascularisation with only a minor occlusion at the ostium of the anterior tibial artery over a portion of a several millimeters. Myonecrosis in the anterolateral and superficial posterior compartment of the left lower leg was seen, suggesting a compartment syndrome (Fig. 1). Furthermore, the scan showed a larger DVT in the right (contralateral) popliteal vein and superficial femoral vein.

Transthoracic echocardiography (TTE) on day 4 postoperatively revealed pulmonary hypertension and right heart failure with strong dilation of the right atrium and right ventricle and moderate dilation of the pulmonary artery, the right ventricular free wall was hypokinetic, and the ventricular septum showed a dyskinetic contraction pattern (early systolic anterior motion) and an eccentric position in systole and diastole (bulging) indicating right ventricular pressure overload with diminished systolic function. Doppler examination revealed a grade $2 / 4$ tricuspid regurgitation with a calculated transtricuspid pressure gradient of $42 \mathrm{~mm} \mathrm{Hg}$. There was a moderate distension of the inferior vena cava with diminished respiratory variation $(<50 \%)$ consistent with an elevated central venous pressure. The left ventricle and left atrium showed normal dimensions and dynamics. There was no thrombus, no left-sided valve pathology nor pericardial effusion (Fig. 2). Following these findings, a thoracic CT-angiogram was performed and revealed the presence of massive bilateral pulmonary embolism in the left lower and proximal in the upper right lobe, middle right lobe and lower right lobe arteries (Fig. 3). This was confirmed by a perfusion scan, clearly showing the multiple segmentary and subsegmentary defect images involving all lobes. Transesophageal echocardiography (TEE) with colour flow mapping (CFM) and administration of IV contrast (agitated saline) (CE) demonstrated a PFO with bidirectional shunt, a small left-to-right shunt (CFM) as shown in Figure 4 and a spontaneous right-to-left shunt (CE). Echocardiographic and CT-angiographic findings were consistent with the diagnosis of acute corpulmonale due to a DVT with massive pulmonary embolism and paradoxical embolism via a PFO. Right heart failure with elevation of right atrial pressure produced a continuous right-to-left shunt, facilitating paradoxical embolization leading to acute arterial occlusion of the left superficial and deep femoral artery.

On day 8 postoperatively, thrombophilia screening was performed which identified an activated protein $\mathrm{C}$ resistance deficiency of $1.5 \%$ (normal value $2.10-3.20 \%$ ). DNA analysis showed that this patient was carrier of the heterozygous factor V Leiden mutation (FVR506Q).

Electromyographic (EMG) investigation on day 9 showed a combination of severe peroneal and a less severe tibial neuropathy, with mostly axon loss. Nerve conduction investigation showed absent peroneal and decreased tibial motor nerve conduction as well as absent sural sensory nerve conduction. Needle EMG showed spontaneous activity in peroneal and tibial innervated muscles, which is the earliest manifestation of axonal denervation. An intensive rehabilitation was performed five times a week with focus on electrical stimulation of affected muscles, progressive resisted exercise of ankle dorsal and plantar flexors, range of motion of the ankle, and 


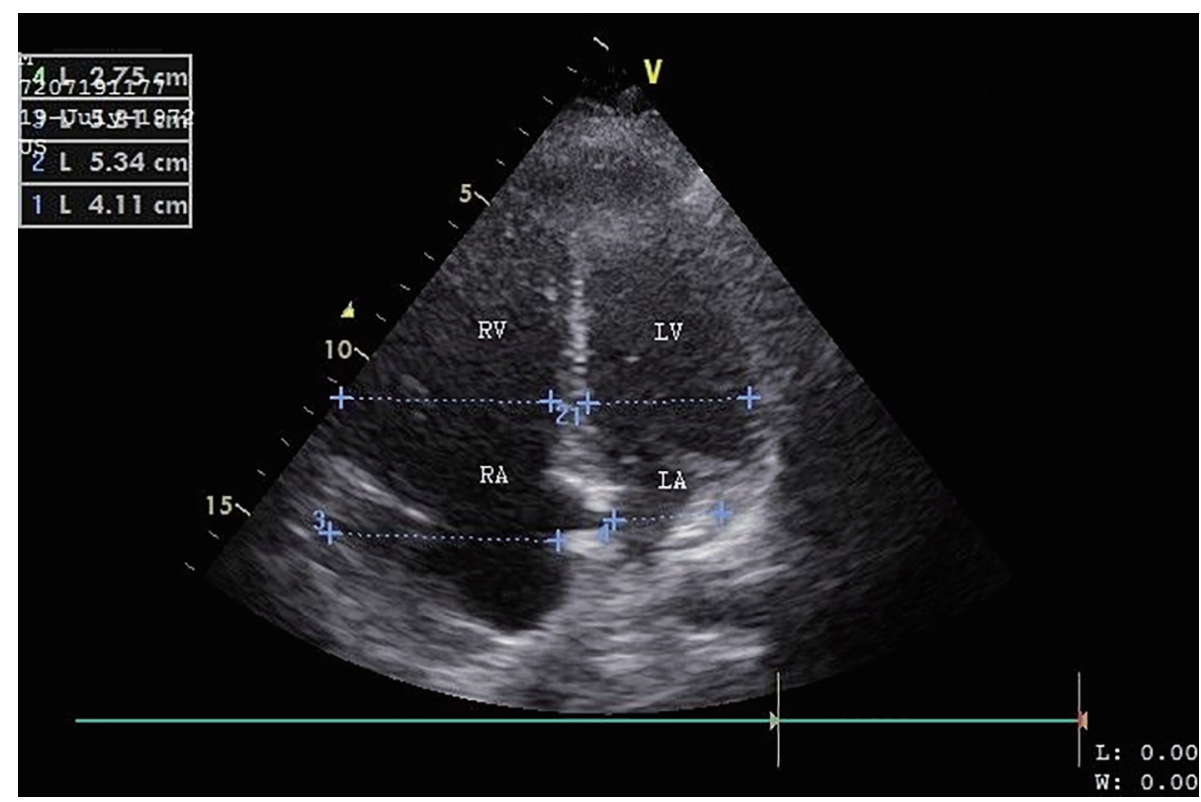

Figure 2. Transthoracic ultrasound (apical four-chamber view) shows a dilated right ventricle and right atrium. RV: right ventricle; RA: right atrium; LV: left ventricle; LA: left atrium.

gait re-education. Because of the foot weakness, walking aid and an ankle-foot orthosis were necessary to reduce fall risk. Our patient was treated with rivaroxaban $20 \mathrm{mg}$ once daily for his venous thromboembolism (VTE). Clinical, ECG and echocardiographic evaluation (TTE) after 8 weeks revealed no residual signs of pulmonary hypertension, and dimensions and
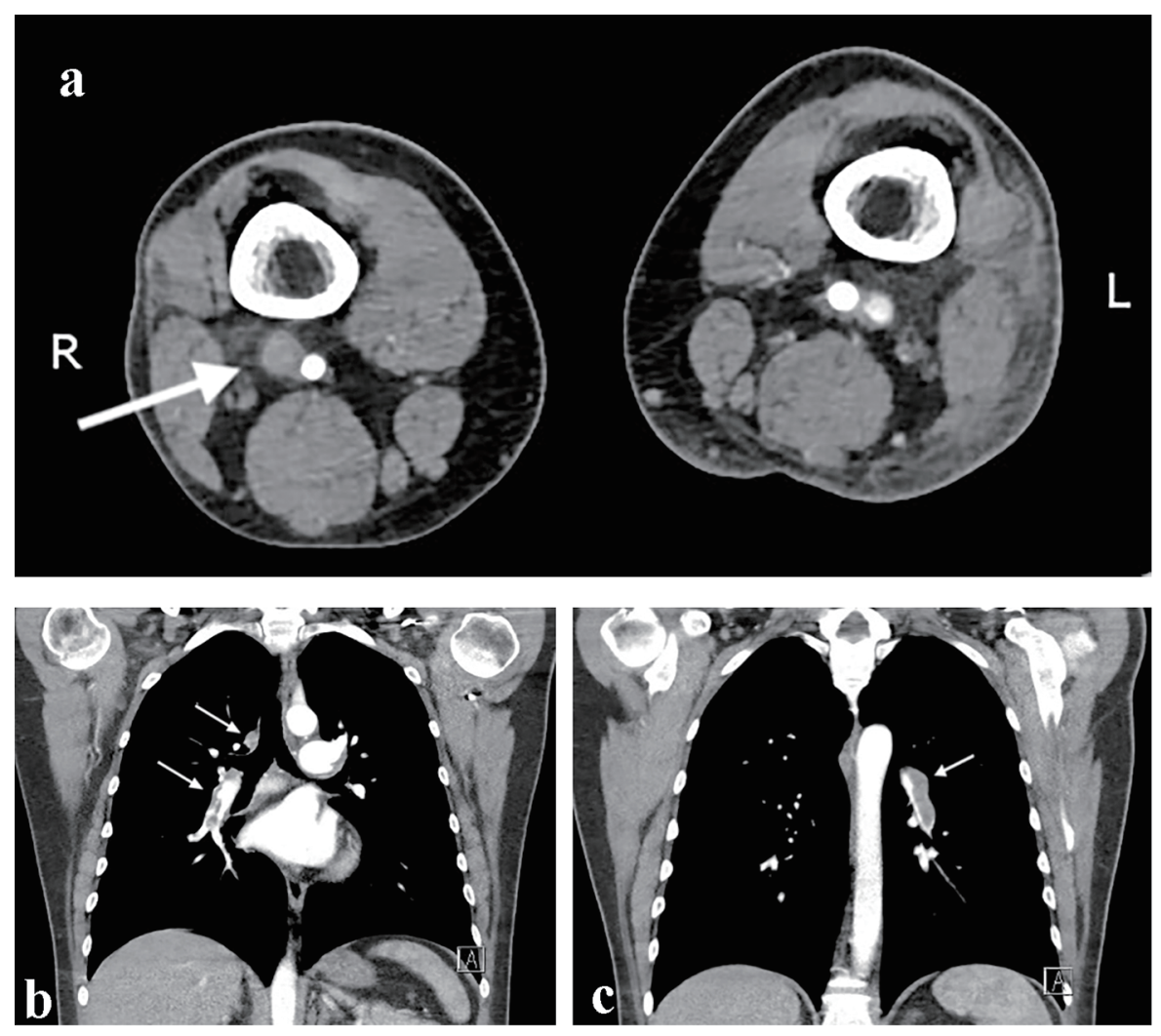

Figure 3. Computed tomography angiography of the abdomen and thorax. (a) The white arrow shows the deep vein thrombosis on the contralateral femoro-popliteal level. (b, c) Bilateral lung embolization. 


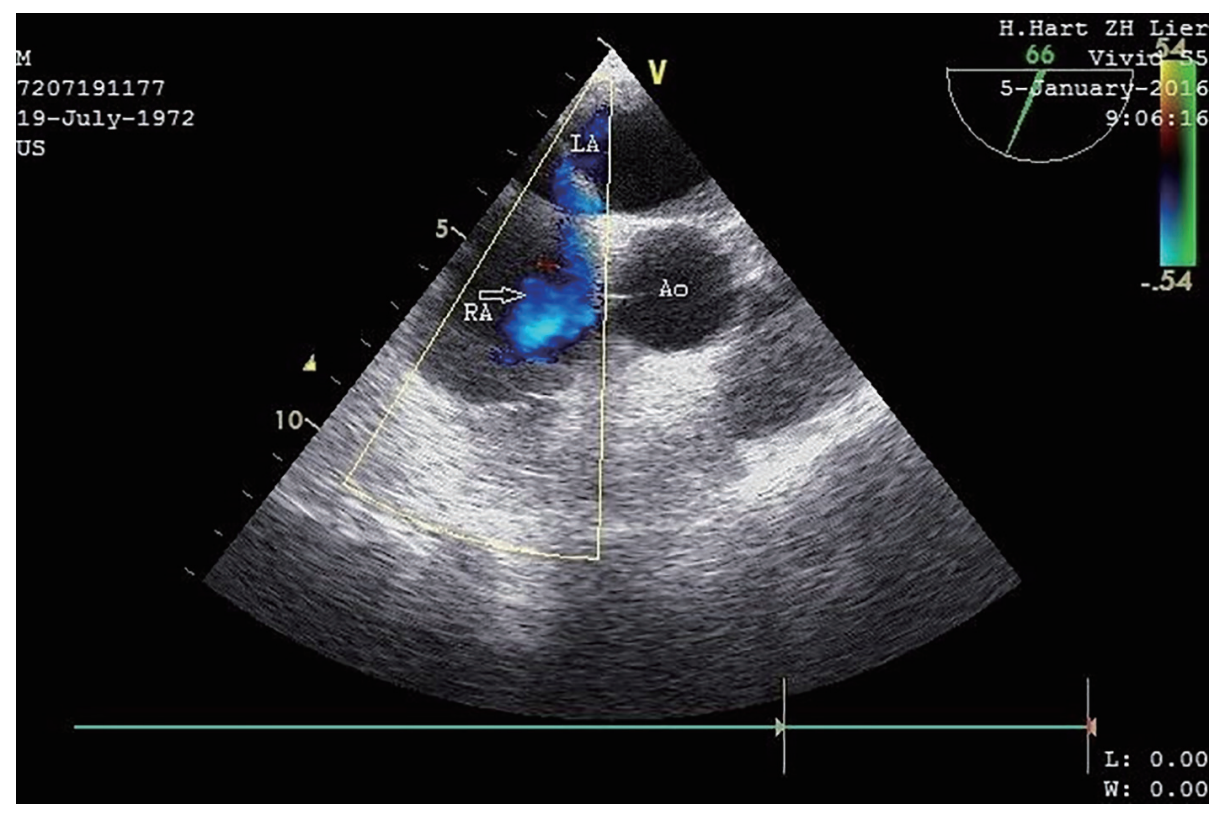

Figure 4. Transesophageal ultrasound with CFM (short-axis view) demonstrates a PFO with a small left-to-right shunt (arrow). IV contrast and Valsalva maneuver also confirmed the presence of a right-left shunt (not recorded). LA: left atrium; RA: right atrium; Ao: proximal ascending aorta.

dynamics of the right ventricle were within normal limits with normal pulmonary artery pressure and right atrial pressure.

At the 5-month follow-up, the patient reported significant improvement in pain, sensation, strength and function. Repeat EMG showed reinnervation potentials and improvement of maximal contraction in muscles innervated by tibial and peroneal nerve, where the latter was not fully restored. Patient was able to walk independently without orthosis and had restarted working. Because of the young age of the patient, the clinical presentation with extensive symptoms and the genetic risk factor, we decided to transfer the patient to a university hospital to perform an endovascular PFO closure after 6 months of oral anticoagulation with rivaroxaban.

\section{Discussion}

A PFO is the most prevalent atrial septum defect and patients often remain asymptomatic. The current gold standard for the diagnosis of a PFO is the use of contrast-enhanced TEE identifying a right-to-left shunting during Valsalva maneuver [7]. A recent meta-analysis described the use of transthoracic echocardiography with harmonic imaging (TTE-HI) as a primary screening test for detecting right-to-left shunts because of its non-invasiveness and high accuracy [8]. Harmonic imaging is based on the principle of receiving double the emitted ultrasound frequency [9]. TTE-HI has a sensitivity and specificity of $90.5 \%$ and $92.6 \%$, respectively. TTE-HI produces the highest sensitivity and specificity when agitated saline with blood is injected before the provocation maneuver, and positive cardiac right-to-left shunt (RLS) is considered by appearance of microbubbles in the left atrium within three cardiac cycles [8]. A major advantage of TTE is the fact that complications such as pulmonary embolism or pulmonary hypertension can be visualized.

A recent meta-analysis of 21 randomized controlled trials confirmed that PFO is associated with 3.4-fold migraine with aura but that there is no association with migraine without aura [10]. Different pathophysiological mechanisms have been postulated like right-to-left shunting of small subclinical emboli and metabolites bypassing the pulmonary system and causing irritation of the trigeminal nerve or an increased platelet activation and aggregation in response to serotonin could possibly trigger migraine and aura (normally serotonin is metabolized in the pulmonary circulation by the lung monoamine oxidase) $[11,12]$. A 2010 meta-analysis concluded that closing the PFO could cure all the migraine symptoms in $46 \%$ of the patients and reduce the symptoms in about $83 \%$ of the patients [13]. Because of the remaining uncertainties, it is still not recommended to use PFO closure as a treatment for migraine with aura and more research is necessary to declare the role between migraine and PFO and the risk to develop other complications, e.g., a paradoxical embolism as described in our case.

ACS mostly develops after trauma, but may also occur after non-traumatic causes like ischemia-reperfusion injury, thrombosis or embolectomy. An increase in intra-compartmental pressure compromises the circulation of the muscles and nerves, which requires prompt surgical decompression. Evidently, the site of neurologic symptoms by ACS is dependent on the affected compartment. As seen in our case, increased pressure in the anterior compartment of the lower leg can cause drop foot by ischemia of deep peroneal nerve and/or most of the ankle dorsiflexors. Raised pressure in the (superficial or deep) posterior compartment can lead to weak ankle plantar flexion, since these compartments include the tibial nerve and the muscles for ankle plantar flexion (27). Electromyography 
in ACS with neurological symptoms is interesting in two aspects. First of all, location, severity and pathophysiologic nature of nerve injury is essential in patient management. Axonal nerve injury (which is mostly the case in ACS) has a relatively poor prognosis compared to segmental demyelination. Partial neuropathy will recover faster than complete lesions. Furthermore, EMG can differentiate between nerve ischemia and primary muscle necrosis as the cause of foot weakness [14].

About $50 \%$ of the patients presenting with a VTE have an underlying thrombophilia which can be inherited or acquired $[15,16]$. Inherited thrombophilia syndromes affect the procoagulant or the anticoagulant (protein $\mathrm{C}$, protein $\mathrm{S}$ or antithrombin) pathway. These syndromes include those with a gain of function mutation as for example the factor $\mathrm{V}$ Leiden- and the prothrombin G20210A mutation as found in the genetic screening of our patient. Factor $\mathrm{V}$ Leiden mutation refers to a specific point mutation making it less susceptible for cleavage by protein $\mathrm{C}$, by this way promoting clot forming. A prothrombin G20210A mutation leads to higher levels of this protein promoting clot forming [15].

Guidelines published in 2010, in the British Journal of Hematology, showed that testing for heritable thrombophilia is only indicated in patients for whom this would affect clinical management. This includes people below 40 years of age presenting with an unprovoked VTE and those with more than two other symptomatic family members [15, 17]. Routine screening of every patient with VTE is therefore not indicated. Two very large prospective studies showed that even if prothrombotic abnormalities were found on screening, this had little effect on the clinical practice with regard to prophylactic strategies $[18,19]$.

Once a genetic screening has been performed and turned out to be positive for a prothrombotic mutation, does this mean that relatives have to be tested and treated? A large populationbased case-control study showed that only a positive family history increased the risk of venous thrombosis more than twofold, regardless of the risk factors or genetic mutation precipitating the thrombosis [20]. Many studies were performed, testing the VTE risk for asymptomatic relatives once a hereditary thrombophilia was found in a patient presenting with VTE. These studies showed that the risk for the relatives was generally very low even during high risk situations [15]. If we focus on factor $\mathrm{V}$ Leiden in particular, we can use the results of a retrospective study published in 1998 by Middeldorp et al showing that the first-degree relative of respectively a symptomatic heterozygous and homozygous has an absolute annual risk for a first episode of VTE of only $0.45 \%$ (CI: $0.28-0.61 \%$ ) and $0.40 \%$ (CI: $0.01-0.90 \%$ ). This incidence did not differ from the incidence of a control population of relatives of patients presenting with a VTE without the factor V Leiden mutation [21]. These data were confirmed in a systematic review [22], but further research is still necessary to determine more specific data regarding incidence in different populations. Currently, testing of relatives is not recommended.

\section{Conflict of Interest}

None.

\section{References}

1. Georgopoulos SE, Chronopoulos A, Dervisis KI, Arvanitis DP. Paradoxical embolism. An old but, paradoxically, under-estimated problem. J Cardiovasc Surg (Torino). 2001;42(5):675-677.

2. Hagen PT, Scholz DG, Edwards WD. Incidence and size of patent foramen ovale during the first 10 decades of life: an autopsy study of 965 normal hearts. Mayo Clin Proc. 1984;59(1):17-20.

3. Meissner I, Whisnant JP, Khandheria BK, Spittell PC, O'Fallon WM, Pascoe RD, Enriquez-Sarano M, et al. Prevalence of potential risk factors for stroke assessed by transesophageal echocardiography and carotid ultrasonography: the SPARC study. Stroke Prevention: Assessment of Risk in a Community. Mayo Clin Proc. 1999;74(9):862-869.

4. Bruch L, Parsi A, Grad MO, Rux S, Burmeister T, Krebs H, Kleber FX. Transcatheter closure of interatrial communications for secondary prevention of paradoxical embolism: single-center experience. Circulation. 2002;105(24):2845-2848.

5. Windecker S, Wahl A, Chatterjee T, Garachemani A, Eberli FR, Seiler C, Meier B. Percutaneous closure of patent foramen ovale in patients with paradoxical embolism: long-term risk of recurrent thromboembolic events. Circulation. 2000;101(8):893-898.

6. Konstantinides S, Geibel A, Kasper W, Olschewski M, Blumel L, Just H. Patent foramen ovale is an important predictor of adverse outcome in patients with major pulmonary embolism. Circulation. 1998;97(19):1946-1951.

7. Seiler C. How should we assess patent foramen ovale? Heart. 2004;90(11):1245-1247.

8. Mojadidi MK, Winoker JS, Roberts SC, Msaouel P, Zaman MO, Gevorgyan R, Tobis JM. Accuracy of conventional transthoracic echocardiography for the diagnosis of intracardiac right-to-left shunt: a meta-analysis of prospective studies. Echocardiography. 2014;31(9):10361048 .

9. Kuhl HP, Hoffmann R, Merx MW, Franke A, Klotzsch C, Lepper W, Reineke T, et al. Transthoracic echocardiography using second harmonic imaging: diagnostic alternative to transesophageal echocardiography for the detection of atrial right to left shunt in patients with cerebral embolic events. J Am Coll Cardiol. 1999;34(6):18231830.

10. Takagi H, Umemoto T, Group A. A meta-analysis of casecontrol studies of the association of migraine and patent foramen ovale. J Cardiol. 2016;67(6):493-503.

11. Sharma A, Gheewala N, Silver P. Role of patent foramen ovale in migraine etiology and treatment: a review. Echocardiography. 2011;28(8):913-917.

12. Zeller JA, Frahm K, Baron R, Stingele R, Deuschl G. Platelet-leukocyte interaction and platelet activation in migraine: a link to ischemic stroke? J Neurol Neurosurg Psychiatry. 2004;75(7):984-987.

13. Butera G, Biondi-Zoccai GG, Carminati M, Caputi L, Usai S, Bussone G, Meola G, et al. Systematic review and 
meta-analysis of currently available clinical evidence on migraine and patent foramen ovale percutaneous closure: much ado about nothing? Catheter Cardiovasc Interv. 2010;75(4):494-504.

14. Katirji B. Peroneal neuropathy. Neurol Clin. 1999;17(3):567-591, vii.

15. Middeldorp S. Evidence-based approach to thrombophilia testing. J Thromb Thrombolysis. 2011;31(3):275-281.

16. Kwon AJ, Roshal M, DeSancho MT. Clinical adherence to thrombophilia screening guidelines at a major tertiary care hospital. J Thromb Haemost. 2016;14(5):982-986.

17. Baglin T, Gray E, Greaves M, Hunt BJ, Keeling D, Machin $\mathrm{S}$, Mackie I, et al. Clinical guidelines for testing for heritable thrombophilia. Br J Haematol. 2010;149(2):209220.

18. Baglin T, Luddington R, Brown K, Baglin C. Incidence of recurrent venous thromboembolism in relation to clinical and thrombophilic risk factors: prospective cohort study. Lancet. 2003;362(9383):523-526.
19. Christiansen SC, Cannegieter SC, Koster T, Vandenbroucke JP, Rosendaal FR. Thrombophilia, clinical factors, and recurrent venous thrombotic events. JAMA. 2005;293(19):2352-2361.

20. Bezemer ID, van der Meer FJ, Eikenboom JC, Rosendaal FR, Doggen CJ. The value of family history as a risk indicator for venous thrombosis. Arch Intern Med. 2009;169(6):610-615.

21. Middeldorp S, Henkens CM, Koopman MM, van Pampus EC, Hamulyak K, van der Meer J, Prins MH, et al. The incidence of venous thromboembolism in family members of patients with factor $\mathrm{V}$ Leiden mutation and venous thrombosis. Ann Intern Med. 1998;128(1):15-20.

22. Segal JB, Brotman DJ, Necochea AJ, Emadi A, Samal L, Wilson LM, Crim MT, et al. Predictive value of factor V Leiden and prothrombin G20210A in adults with venous thromboembolism and in family members of those with a mutation: a systematic review. JAMA. 2009;301(23):2472-2485. 